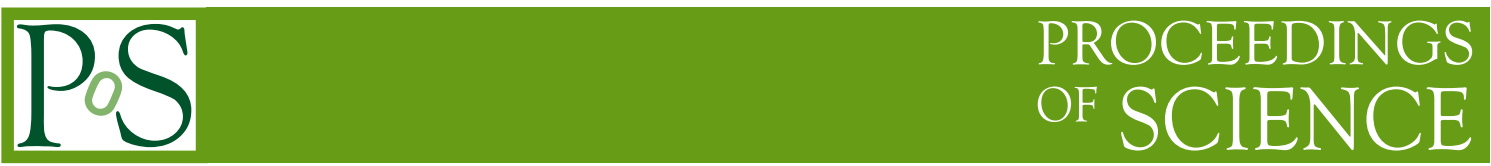

\title{
W/Z+jets and W/Z+HF-jets production at ATLAS
}

\author{
Camilla Vittori, on behalf of the ATLAS Collaboration \\ Università di Bologna and INFN \\ E-mail: camilla.vittori@cern.ch
}

\begin{abstract}
The production of jets in association with a $\mathrm{W}$ or a $\mathrm{Z}$ boson in proton-proton collisions is an important process to study QCD in multi-scale environments. Moreover, measurements of W/Z boson production associated with heavy-flavour (HF) quarks provide important experimental constraints to improve the theoretical description of these processes, as the uncertainties in the current predictions are larger than the ones obtained in the inclusive case. The detailed knowledge of the production of jets associated with electroweak gauge bosons is also a key element for the understanding of Higgs initiated processes and Beyond Standard Model searches, as they represent an important background in these measurements. Results for the differential production cross sections for W/Z+jets and W/Z+HF-jets with respect to several kinematics variables measured by ATLAS in proton-proton collisions at centre-of-mass energies of 7 and $13 \mathrm{TeV}$ are presented and compared to high-order QCD calculations and recent Monte Carlo simulations.
\end{abstract}

EPS-HEP 2017, European Physical Society conference on High Energy Physics 5-12 July 2017

Venice, Italy 


\section{Introduction}

The measurement of the $\mathrm{Z}$ boson production in association with jets ( $\mathrm{Z}+\mathrm{jets})$ benefit from the high production rate and the clean leptonic final state of the $\mathrm{Z}$ boson. This allows to perform high precision measurements, to test perturbative QCD (pQCD) in a multi-scale environment and to compare with several Monte Carlo (MC) predictions. Such processes also constitute a nonnegligible background for studies of the Higgs boson and in searches of new phenomena with high jet multiplicity final states. In this report the measurement of the $Z+j$ ets differential cross section at $\sqrt{s}=13 \mathrm{TeV}$ is presented [1]. The cross section is measured as a function of the jets multiplicity $N_{\text {jets }}$ and of jet kinematic variables for different jet multiplicities.

\section{Data and Monte Carlo simulations}

The dataset used in this analysis was collected by ATLAS at the centre of mass energy $\sqrt{s}=$ $13 \mathrm{TeV}$ and corresponds to an integrated luminosity of $3.16 \mathrm{fb}^{-1}$.

Monte Carlo generators are used to unfold the data to the particle level, to compare with the unfolded data distributions and to estimate most of the background events. In the following, a summary of Monte Carlo generators used to simulate signal samples is given; for background samples a full description can be found in [1]. SHERPA 2.2 is the main generator used for the signal production, where the matrix element (ME) is calculated for up to two additional partons at NLO (up to four partons at LO) and matched with parton shower(PS). The MADGRAPH_aMC@NLO generator with the LO ME (up to four partons) and the CKKWL merging scheme for PS (MG5_aMC@NLO CKKWL) is used in the estimation of systematic variations; a MG5_aMC@NLO sample with LO ME (up to two partons) with the FXFX merging scheme for PS (MG5_aMC@NLO FXFX) is employed in the comparison with the unfolded cross sections.

The measured cross sections are also compared to predictions from Alpgen with a LO ME (up to five partons) interfaced with PYTHIA 6 for PS. Parton-level fixed-order predictions are calculated by the BLACKHAT+SHERPA collaboration for the production of the $\mathrm{Z}$ bosons with up to four partons. Moreover calculations at NNLO QCD with a new subtraction technique based on $\mathrm{N}$-jettiness $\left(\mathrm{Z}+\geq 1\right.$ jet $\mathrm{N}_{\text {jetti }}$ ) is used for comparison [1].

\section{Analysis Selection}

Electron and muon events are selected using specific criteria, such as $p_{T}^{l e p}>25 \mathrm{GeV}$ in the region $\left|\eta^{l e p}\right|<2.47$ (excluding the crack region) and $|\eta|<2.4$ respectively [1]. Jets are reconstructed using the anti- $\mathrm{k}_{T}$ algorithm with radius parameter 0.4 , requiring $p_{T}>30 \mathrm{GeV}$ and $|y|<2.5$.

Contributions from $\mathrm{W}+\mathrm{jets}$, diboson and top background components are estimated using Monte Carlo samples. The multijet background is estimated through a data driven technique in enriched regions, constructed by loosening the lepton identification and isolation [1]. Templates are built from the dilepton invariant mass distribution and then normalised to events passing the $\mathrm{Z}+\geq 1$ jet signal selection through a log-likelihood fit. The overall purity of the selection ranges from $99 \%$ in the $\mathrm{Z}+\geq 1$ jet sample to $80-85 \%$ in the $\geq 7$ jets. The dominant background component comes from $t \bar{t}$ production, whose contribution increases from $\sim 1$ to $12 \%$ at high jet multiplicities, as shown in Fig.1a. 


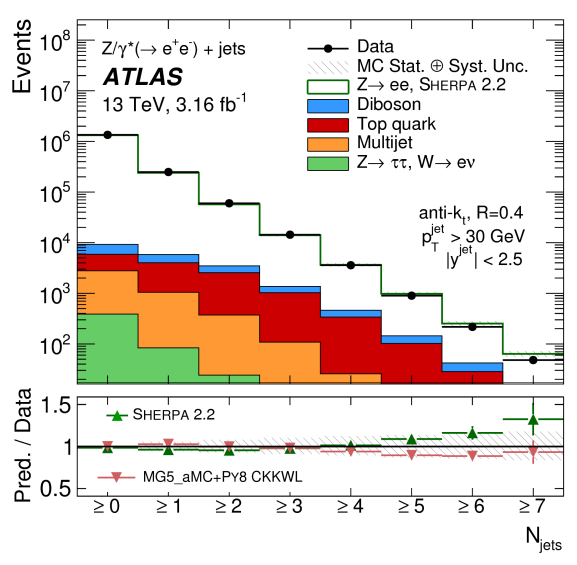

(a)

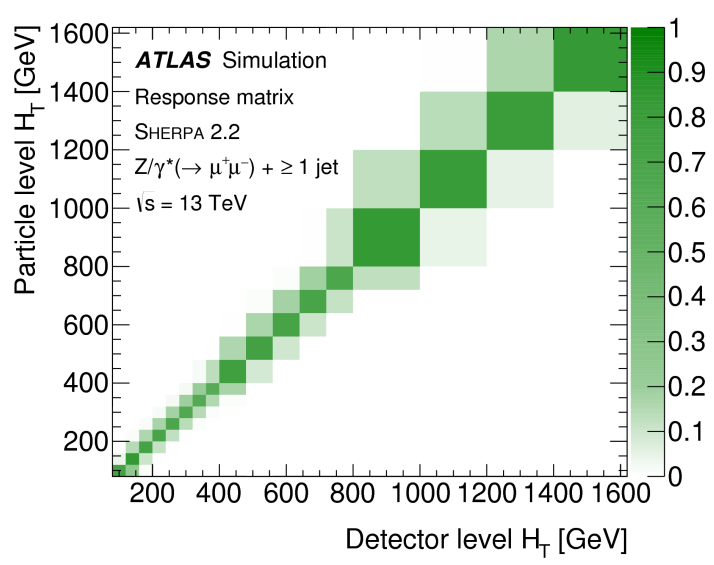

(b)

Figure 1: (a) Inclusive jet multiplicity distribution in the $\mathrm{Z}\left(\rightarrow \mathrm{e}^{+} \mathrm{e}^{-}\right)+$jets channel. (b) Unfolding response matrix corresponding to the $\mathrm{H}_{T}$ for the $\mathrm{Z}\left(\rightarrow \mu^{+} \mu^{-}\right)+$jets events [1].

\section{Unfolding Procedure}

Cross section measurements are performed in the fiducial acceptance region defined by the following requirements:

- $p_{T}^{l}>25 \mathrm{GeV},\left|\eta^{l e p}\right|<2.5$;

- $p_{T}^{\text {jet }}>30 \mathrm{GeV},\left|y^{\text {jet }}\right|<2.5$ and $\Delta R(l$, jet $)>0.4$;

- $71<m_{l l}<111 \mathrm{GeV}$.

Cross sections are defined at particle level, corresponding to dressed leptons from the $\mathrm{Z}$ boson, as a function of the experimental observables $N_{j e t s}, p_{T}^{j e t}, y^{j e t}, \Delta \phi_{j j}, M_{j j}$ and $H_{T}$ (scalar sum of the $p_{T}$ of all final state objects). A variable-width binning of these variables is used, chose in order the purity to be at least $50 \%$ in each bin and the statistical uncertainty to remain below $10 \%$. An iterative Bayesian procedure is used to unfold the measured distribution at particle level, in order to correct for detector inefficiencies and resolutions. This technique updates the initial estimators for the generated distribution (prior) in consecutive steps using the Bayes' theorem to derive the response matrix. Fig. $1 \mathrm{~b}$ shows the response matrix obtained after the application of the unfolding procedure on the $\mathrm{H}_{T}$ distribution. Systematics on the unfolding take into account the limited statistics, the hadronization modelling, the migrations into other kinematic distributions and the finite bin width. An overall uncertainty of $\sim 3 \%$ is associated to the unfolding procedure [1].

\section{Results}

The cross sections measurements have been performed separately in the electron and muon channel and then combined taking into account the correlations between the two modes. Propagated through the unfolding via the migration matrices, the largest contribution to the uncertainties 

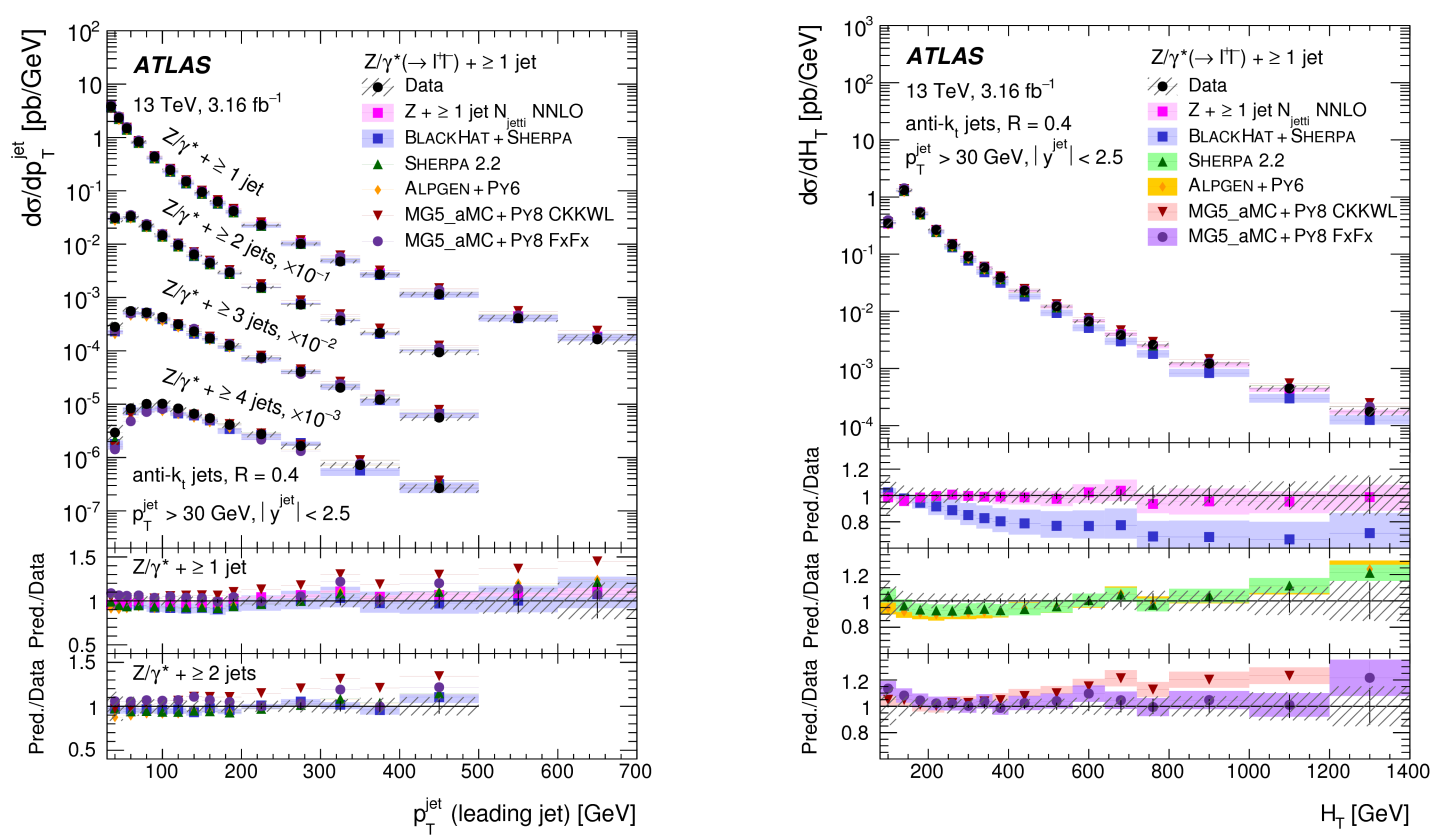

Figure 2: Measured differential cross section as a function of the leading jet $p_{T}$ (left) and $H_{T}$ (right) [1].

is given by jet energy scale ( $8 \%$ in $\mathrm{Z}+\geq 1$ jet up to $25 \%$ in $\mathrm{Z}+\geq 7$ jets).

The combined cross section are compared to the predictions listed in Section 2. In particular, the $p_{T}^{\text {jet }}$ is a fundamental observable for the $\mathrm{Z}+$ jets processes, since it probes $\mathrm{pQCD}$ over a wide range of scales. The leading-jet $p_{T}$ distribution in inclusive $\mathrm{Z}+\geq 1,2,3,4$ jet events is shown in Fig.2 (left) and ranges up to $700 \mathrm{GeV}$. While the LO generator MG5_aMC+PY8 CKKWL models a too hard $p_{T}^{\text {jet }}$ spectrum, NLO predictions from BLACKHAT+SHERPA, SHERPA 2.2 and MG5_aMC+PY8 FXFX are in agreement with the measured cross sections over the full range. ALPGEN+PY6 also shows a good agreement with the measured data. The $\mathrm{Z}+\geq 1$ jet NNLO prediction well models the spectrum for $\mathrm{Z}+\geq 1$ jet.

The differential cross section as a function of $H_{T}$ for $Z+\geq 1$ jet events is shown in Fig.2 (right). Predictions from SHERPA 2.2, ALPGEN+PY6, MG5_aMC+PY8 FXFX well describe the data, while MG5_aMC+PY8 CKKWL overestimates the contribution at large value of $H_{T}$. Fixed order NLO predictions from BLACKHAT+SHERPA underestimate the cross section for values of $H_{T}>300$ $\mathrm{GeV}$, due to the missing contributions from events with higher parton multiplicities. Agreement is recovered by adding higher order in $\mathrm{pQCD}$, as demonstrated by the good description of $H_{T}$ by $\mathrm{Z}+\geq 1$ jet $\mathrm{N}_{\text {jetti }}$ NNLO.

\section{References}

[1] ATLAS Collaboration, Measurements of the production cross section of a $Z$ boson in association with jets in pp collisions at $\sqrt{s}=13$ TeV with the ATLAS detector, Eur. Phys. J. C77 (2017) 361. 